\title{
Liderança em enfermagem hospitalar: Percepção de enfermeiros gestores
}

\author{
Leadership in hospital nursing: Perception of managing nurses \\ Liderazgo en enfermería hospitalaria: Percepción de las enfermeras directivas
}

Recebido: 05/07/2021 | Revisado: 14/07/2021 | Aceito: 20/07/2021 | Publicado: 28/07/2021

\author{
Rafael Gomes \\ ORCID: https://orcid.org/0000-0002-4852-5813 \\ Centro Universitário INTA, Brasil \\ E-mail: rafael.gomesgomes@outlook.com \\ Adriano da Costa Belarmino \\ ORCID: https://orcid.org/0000-0003-4401-9478 \\ Universidade Estadual do Ceará, Brasil \\ E-mail:adrian_belarmino@hotmail.com \\ Cleiton Machado Pinho \\ ORCID: https://orcid.org/0000-0002-8478-4804 \\ Secretaria Municipal de Saúde do Ceará, Brasil \\ E-mail: cleiton.pinho@gmail.com \\ Patrícia de Oliveira Bastos \\ ORCID: https://orcid.org/0000-0002-7052-8017 \\ Universidade Estadual do Ceará, Brasil \\ E-mail: nurse.paty9@gmail.com \\ Antonia Abigail do Nascimento Cavalcante \\ ORCID: https://orcid.org/0000-0001-5172-6088 \\ Centro Universitário INTA, Brasil \\ E-mail:abigail.nascimento123@gmail.com \\ Larissa Cunha Alves \\ ORCID: https://orcid.org/0000-0001-6173-7549 \\ Centro Universitário INTA, Brasil \\ E-mail: larissacalves@hotmail.com
}

\section{Resumo}

Introdução: Liderança em enfermagem mostra-se atualmente como elemento imprescindível para o desenvolvimento de gestão em equipes de saúde. Objetivo: conhecer a percepção dos enfermeiros sobre a liderança como instrumento no seu processo de trabalho. Método: Trata-se de pesquisa descritiva e qualitativa realizada em hospital de alta complexidade do Ceará. Os participantes do estudo foram dez enfermeiros gestores que atuam nas coordenações de serviços assistenciais. Entrevistas semiestruturadas com roteiro foram utilizadas para coleta de informações efetuadas mês de junho de 2017 e os depoimentos foram submetidos à análise de conteúdo de Bardin. Resultados: Quatro categorias emergiram: Liderança e as relações interpessoais no Trabalho; Fragilidades e potencialidades enfrentadas pelo gestor; Fatores que motivam os colaboradores no ambiente de trabalho; Fragilidade na formação acadêmica em gestão. Os resultados sinalizam que é característica do enfermeiro exercer o papel de líder, sendo fundamental na organização do serviço, em que habilidades potentes de escuta, diálogo e gestão participativa são essenciais. Considerações finais: É importante a liderança da enfermagem nos serviços para que haja cooperação dos colaboradores da organização para cumprimento de metas e alcance na qualidade do cuidado aos pacientes.
\end{abstract}

Palavras-chave: Liderança; Enfermagem; Cuidado; Gestão em saúde; Equipe de saúde.

\begin{abstract}
Introduction: Leadership in nursing is currently shown as an essential element for the development of management in health teams. Objective: to know the nurses' perception of leadership as an instrument in their work process. Method: This is a descriptive and qualitative research carried out in a high complexity hospital in Ceará. The study participants were ten nurse managers who work in the coordination of care services. Semi-structured interviews with a script were used to collect information carried out in June 2017 and the testimonies were submitted to Bardin's content analysis. Results: Four categories emerged: Leadership and interpersonal relationships at work; Weaknesses and potentials faced by the manager; Factors that motivate employees in the workplace; Weakness in academic management training. The results indicate that it is characteristic of nurses to play the role of leader, being essential in the organization of the service, in which powerful listening skills, dialogue and participatory management are essential. Final considerations: The leadership of nursing in services is important so that there is cooperation of the organization's employees to achieve goals and reach the quality of patient care.
\end{abstract}

Keywords: Leadership; Nursing; Care; Health Management; Health team. 


\begin{abstract}
Resumen
Introducción: El liderazgo en enfermería se muestra actualmente como un elemento fundamental para el desarrollo de la gestión en los equipos de salud. Objetivo: conocer la percepción de las enfermeras sobre el liderazgo como un instrumento en su proceso de trabajo. Método: Se trata de una investigación descriptiva y cualitativa realizada en un hospital de alta complejidad en Ceará. Los participantes del estudio fueron diez enfermeras gestoras que trabajan en la coordinación de los servicios asistenciales. Para la recogida de información se utilizaron entrevistas semiestructuradas con guión realizadas en junio de 2017 y los testimonios fueron sometidos al análisis de contenido de Bardin. Resultados: Surgieron cuatro categorías: liderazgo y relaciones interpersonales en el trabajo; Debilidades y potenciales que enfrenta el gerente; Factores que motivan a los empleados en el lugar de trabajo; Debilidad en la formación en gestión académica. Los resultados indican que es característico del enfermero desempeñar el papel de líder, siendo fundamental en la organización del servicio, en el que son fundamentales la poderosa capacidad de escucha, el diálogo y la gestión participativa. Consideraciones finales: El liderazgo de enfermería en los servicios es importante para que exista la cooperación de los empleados de la organización para lograr las metas y alcanzar la calidad de la atención al paciente.
\end{abstract}

Palabras clave: Liderazgo; Enfermería; Precaución; Manejo de la salud; Equipo de salud.

\title{
1. Introdução
}

A liderança em organizações de saúde possui atualmente papel de destaque diante das mudanças globais e promoção da saúde populacional $(Y i, 2018)$. Estratégias mundiais, em inúmeros cenários e especialmente em países de baixa e média renda, tem defendido a liderança como estratégia principal para garantir serviços de qualidade e eficazes, assim como reformulações em sistemas de saúde (Nanyonga et al., 2020).

Neste cenário, o enfermeiro configura-se como um dos principais provedores de liderança em equipes de saúde, estando inserido em inúmeros espaços e níveis de atenção em saúde, e constituindo-se em coordenador e gestor de equipes e serviços (Bitencourt et al., 2020).

A liderança em enfermagem é descrita como a habilidade de liderar a equipe, que garanta que os integrantes em conjunto consigam alcançar objetivos compartilhados, em que garantir as necessidades em saúde de pacientes, famílias e comunidades seja alcançado (Moura et al., 2019).

Além disso, em uma sociedade altamente globalizada e de mercado competitivo, a liderança é uma competência na prática profissional do enfermeiro indispensável para o desenvolvimento de suas atividades, criando ambientes positivos ou negativos dependendo do tipo de liderança adotado pelo enfermeiro, inspiração e estimulo para equipe na introdução de inovações e mudanças, assim como antecipação de problemas e adoção de medidas corretivas que promovam satisfação dos profissionais da equipe (Moura et al, 2020).

No entanto, fatores como por exemplo o ambiente organizacional podem afetar o modo de liderar do enfermeiro. No mundo atual, relacionado ao mercado de trabalho e exigências crescentes de produtividade e qualidade do trabalho, exige-se qualificação relacionada a modelos formativos e gestores baseados em competências profissionais. Entre as características de um líder qualificado destaca-se: visão, competência, habilidades comunicativas, tomada de decisão, planejamento, capacidade resolutiva relativo a problemas, bom relacionamento e estabilidade emocional (Carrara et al., 2017).

Ademais, a formação de enfermeiros-líderes tem tido destaque nas graduações em saúde para atuação em equipes multiprofissionais e em organizações de saúde (moura et al., 2019). Internacionalmente, tem sido tema de estudos em inúmeros países como Coreia, Uganda, Brasil e Espanha, entre outros (Yi, 2018; Nanyonga et al., 2020; Bitencout et al., 2020; Moura et al., 2019; Estalella et al., 2021).

Dadas as singularidades nas práticas de cuidado, pela individualidade de cada profissional e de cada usuário, espera-se do enfermeiro o adequado gerenciamento dos processos e necessidades de saúde, de maneira a exercer sua capacidade de liderança e de tomada de decisões, com criatividade, inovação e visão ampliada do seu fazer (Ferreira et al., 2016)

Enquanto alguns conseguem empreender novas ações, tomar iniciativas, buscar novas formas de agir e gerar trocas construtivas no seu fazer, outros se conformam com a situação tal como se apresenta, limitando-se à zona de conforto e a uma 
rotina de obrigações, não indo além do meramente prescrito. Fragilidades como conflitos na equipe de saúde, falta de iniciativa, falta de engajamento na cultura organizacional e na tomada de decisão nos processos de trabalho são importantes fatores que impactam no cuidado e na assistência. Entretanto, tem-se observado diferentes modos de trabalhar entre profissionais que compartilham o mesmo ambiente laboral, com responsabilidades idênticas e nas mesmas circunstâncias (Ferreira et al., 2016).

Diante disso, questionou-se como o enfermeiro percebe os aspectos de liderança como instrumento para seu trabalho?

É importante discutir o papel da liderança da enfermagem e rever fragilidades e potencialidades no trabalho em equipe que possibilitem maximizar resultados em saúde, qualidade na assistência e harmonia laboral na equipe de enfermagem e de saúde, objetivando alcançar metas em comum com bons resultados aos pacientes assistidos.

Diante disso, objetivou-se no estudo identificar a percepção dos enfermeiros sobre a liderança como instrumento no processo de trabalho.

\section{Metodologia}

O estudo consiste de pesquisa descritiva com abordagem qualitativa (Minayo, 2014), realizado em um hospital de alta complexidade da região norte do Ceará, Brasil. O hospital é referência para população de 55 municípios da região referida do estado. A instituição possui atendimento de urgência e emergência, unidade de terapia intensiva adulto, clínica médica, assim como serviços de apoio como nutrição, imagem e laboratório, entre outros.

Para participação do estudo foram incluídos os coordenadores de enfermagem da instituição, com um total de 17 coordenadores, que lideram serviços assistenciais. Aceitaram participar da pesquisa dez enfermeiros e utilizou-se para fechamento da amostra, o critério de amostragem por saturação (Minayo, 2014).

A coleta de informações foi realizada no mês de junho do ano de 2017, através de entrevista semiestruturada, utilizando um roteiro com perguntas focalizadas, dividido em três partes: I- caracterização sociodemográfica; II- liderança e as relações interpessoais no trabalho; Parte III - fragilidades e potencialidades enfrentadas pelo gestor.

Explicou-se aos participantes os objetivos do trabalho, e após a concordância, iniciou-se a entrevista. Foi pactuado com os sujeitos o melhor local e horário para a realização da entrevista em local previamente determinado pelos mesmos. Empregou-se gravador digital para gravação e código alfanúmerico com a letra $\mathrm{E}$ e números respectivos para garantir confidencialidade. A duração de cada entevista teve média de 38 minutos. As transcrições duraram dois meses, sendo efetuado por um dos pesquisadores $(\mathrm{RG})$.

Os dados coletados foram submetidos à análise de conteúdo de Bardin, seguindo as etapas de pré-análise, codificação, categorização e interferência. Na primeira fase, organizou-se o material a ser analisado através de leitura flutuante do material. Em seguida, identificou-se códigos nos materiais transcritos. A terceira fase ocorreu a partir do princípio de categorização pela repetição de frases significantes e, por fim, o levantamento do conteúdo subjacente ao que está sendo manifesto, através de interpretação (Minayo 2014).

Os resultados foram analisados e discutidos com a literatura cientifica nacional e internacional sobre liderança em enfermagem para garantir fidedignidade dos achados.

A pesquisa foi submetido à aprovação do Comitê de Ética em Pesquisa (CEP), sob parecer n $n^{\circ}$ 2.111.226, a coleta de dados seguiu os princípios éticos e legais de acordo com a resolução 466 de 2012 (Ministério da Saúde do Brasil, 2012). 


\section{Resultados}

\subsection{Caracterização Sociodemográfica}

Surgiram quatro categorias temáticas após organização: Liderança e as relações interpessoais no trabalho; Fragilidades e potencialidades enfrentadas pelo o gestor; Fatores que motivam os colaboradores no ambiente de trabalho; Fragilidade na formação acadêmica em gestão.

A Tabela 1 descreve as informações dos enfermeiros participantes do estudo:

Tabela 1: Distribuição das características sociodemográficas e profissionais dos participantes. Sobral - Ceará, Brasil, 2017.

\begin{tabular}{lll}
\hline Variáveis & $\mathbf{N}$ & $\mathbf{\%}$ \\
\hline Idade: & 08 & $80 \%$ \\
28 a 33 & 00 & $0 \%$ \\
34 a 39 & 01 & $10 \%$ \\
40 a 45 & 01 & $10 \%$ \\
46 a 51 & & \\
\hline Gênero: & 09 & $90 \%$ \\
Feminino & 01 & $10 \%$ \\
Masculino & & $100 \%$ \\
\hline Categoria: & 10 & $100 \%$ \\
Enfermeiro Especialista & & $30 \%$ \\
\hline Habilidades Acadêmicas: & 10 & \\
Especialistas & 03 & $70 \%$ \\
Mestrado & & $30 \%$ \\
\hline Tempo de exercício profissional: & 07 & $10 \%$ \\
48 à 120 meses & 03 & $10 \%$ \\
132 à 360 meses & & $80 \%$ \\
\hline Tempo de serviço onde trabalha: & 01 & $100 \%$ \\
07 à 11 meses & 01 & $100 \%$ \\
12 à 24 meses & 08 & \\
36 à 48 meses & & \\
\hline Vínculo: & 10 & \\
CLT & 10 & \\
\hline Interesse pelo cargo de liderança: & & \\
Sim & & \\
\hline
\end{tabular}

Fonte: Dados da pesquisa.

No que se refere à idade dos participantes têm uma média de idade de 28 a 51 anos, sendo $80 \%$ na idade de 28 à 33 anos. O sexo predominante entre os entrevistados foi sexo feminino com nove enfermeiras e um enfermeiro. A categoria de entrevistados é de $100 \%$ com especializações, e $30 \%$ com mestrado.

Relativo ao exercício profissional percebe-se um valor de $70 \%$ com tempo de 48 à 120 meses, e de 30\% com tempo de 132 à 360 meses, com a maioria possuindo de quatro a dez anos de profissão.

A seguir, descreve-se as quatro categorias temáticas a partir do processo analítico. 


\subsection{Categoria I: Liderança e as relações interpessoais no trabalho.}

Durante as entrevistas quando os entrevistados foram questionados sobre a resolução de conflitos entre a equipe observou-se, fortemente, a imparcialidade como estratégia de resolução de conflitos.

"Busco ser uma mediadora de conflitos, então assim, nessa gestão de pessoas procuro ouvir os dois lados, não tomar decisões precipitadas e tentar sempre mediar; ouvir o técnico de enfermagem, o enfermeiro que é o líder; sempre permito que o enfermeiro venha se posicionar como líder para também o enfermeiro ser um mediador de conflitos". $(E-3)$

"A gente tem que trabalhar muito a pessoa, a maturidade; a gente tem que colocar sempre como objetivo a filosofia da instituição que é o trabalho em equipe, o respeito, profissionalismo; que apesar das diferenças, apesar dos problemas, a gente precisa entrar aqui como profissional, esquecer os conflitos que existem, e que aqui a gente precisa trabalhar em equipe e a gente precisa funcionar todos como uma equipe pelo paciente; então a gente escuta as queixas, escuta os lados, mas a gente sempre coloca que todos precisam buscar o respeito e trabalhar em equipe". $(E-4)$

Ao observar os depoimentos dos entrevistados, pode-se perceber que a busca do diálogo é uma estratégia utilizada pelo os gestores desta instituição para resolução de conflitos:

"A gente busca resolver através do diálogo, tem que ouvir sempre as partes envolvidas, e a partir disso tirar algo construtivo, sempre na visão construtivista; através do conflito tentar tirar algo positivo para que seja bom para a prestação de serviço, para o convívio da equipe”. (E-7).

"Tento primeiro ouvir, buscar todas as versões, tentando esclarecer o máximo possivel a verdade, buscando o princípio da verdade e da justiça, tentando esclarecer os fatos, e ouvir quem está envolvido sempre na base do diálogo". (E- 8)

"Eu sou muito aberta e gosto muito de conversar com eles, a cada conflito eu gosto de escutar ambas as partes; a gente não pode ser unilateral, e qualquer tipo de conflito tenho que avaliar se vai levar algum tipo de risco ao paciente; nosso maior propósito é a segurança do paciente dentro da instituição; por muitas vezes são conflitos pessoais que com uma boa conversa, uma boa orientação tem como ser resolvido; poucas as vezes tenho que impor algo dentro da equipe, sou muito aberta e é uma equipe muito unida". (E-10)

Em relação ao envolvimento dos profissionais nas decisões que os afetam, podemos perceber que a gestão participativa foi citada como estratégia para envolvimento dos colaboradores nos processos de trabalho, como relatado a seguir:

"Particularmente faço uma gestão bem participativa, realizando reuniões quinzenais, envio material pra eles, eles estudam e depois debatemos sendo bem democrático”. (E-5) 
"Quando tem alguma mudança primeiro tem que abordar a equipe, informar, conversar, discutir o que está acontecendo, ouvir as percepções deles, porque muitas vezes as percepções deles ajudam-nos a resolver conflitos e muitas vezes fazer com que eles reflitam sobre algo e cheguem em uma conclusão juntos". (E-6)

O desenvolvimento de relações interpessoais destaca-se nos relatos, enfocando aspectos particulares na equipe de saúde que influenciam na harmonia da equipe e no desenvolvimento de práticas de saúde. Alguns depoimentos destacam isso:

"A maior valorização é tentar envolvê-los, mostrar que dependem um do outro, que ninguém faz um trabalho individual só e vai ser reconhecido só. Devemos ter um trabalho respeitoso, não precisa ser amigo mas trabalho é trabalho. Atenção é fundamental para o próximo, e o que todos fazem ou a decisão tomada reflete no paciente". (E-5)

Os sujeitos envolvidos nas atividades de cuidado devem participar de forma integrada, apontando os caminhos para constituir uma equipe integrada, partindo da perspectiva do agir comunicativo e, assim, conceber o elo entre os participantes do grupo. Uma das falas destaca as repercussões positivas disso:

"Extremamente gratificante; no setor nós temos uma interação multiprofissional muito forte, e essa relação interpessoal não significa se é particular ou de todo mundo, é respeitar o profissionalismo, respeitar que cada categoria executa a sua ação e a gente poder dialogar; então respeito quando reconheço o trabalho do outro, a importância, assim como também escuto o que ele tem a dizer; a minha competência sobre as minhas ações, escuto aquilo positivamente". (E-7)

Outro quesito evidenciado entre os gestores entrevistados, foi a forma de organização não hierárquica da instituição, exibindo-se claramente o desenvolvimento da liderança democrática como forma de gerir, satisfatoriamente, o serviço de enfermagem. Os relatos a seguir descrevem:

"A gente tem reuniões abertas, que sempre fica em aberto sugestões ou mesmo nas visitas no setor, nas conversas, em que dependendo da viabilidade será acatada; muitas coisas quando identificamos que tem que resolver, tomar uma decisão, a gente conversa e muitas coisas assim a gente acaba ouvindo e colocamos em prática, até porque são eles que estão na ponta, e sabem como fazer e a melhor forma como fazer". (E- 4)

"Eu acho muito positivo, assim quando alguém fala alguma coisa mesmo que seja negativamente, significa que ele está tendo um olhar crítico do que está fazendo, porque pior seria alguém que só faz e não participa; então positivo ou negativo, a gente tem que ouvir pra tentar tirar o melhor da opinião dele, ouvir a crítica e tentar fazer dela o melhor possivel; eu sempre ressalto, que se for construtiva ou que seja negativa, mas sempre eu vou ouvir; a gente sempre tentar diferenciar isso, mas buscar ouvir, mesclar um pouquinho do que tem que ser feito do que eles vêm trazendo pra gente sempre ampliar".(E- 7)

\subsection{Categoria II: Fragilidades e potencialidades enfrentadas pelo o gestor}

Como forma de estimular o desenvolvimento do capital intelectual, os gestores parecem entender a importância da formação profissional, como estratégia de melhorar a qualidade da assistência prestada no serviço. A seguir descreve-se alguns depoimentos: 
"A gente precisa trabalhar muito o processo como pessoa, haver comunicação, que ali é um profissional, mas também é um ser humano, uma pessoa que tem seus vínculos familiares; muitas vezes é um técnico de enfermagem e faz uma graduação em outra área, até mesmo na mesma área, e a gente como coordenador deve incentivar a estar buscando este crescimento desde agora; a gente tem programas, quando eles precisarem ir pra congresso tem a liberação. Podemos organizar a escala pra eles irem pra curso, especialização. Para que ele continue buscando e se aperfeiçoe cada vez mais". (E- 4)

"Sabemos que não somos preparados para gerir pessoas e eu estimulo a todos estudar, a buscar conhecimentos sobre gestão, sobre liderança e em um todo. E o que aprendo tento passar e quando a equipe estar sem gás tento animar". (E- 5)

Desenvolver liderança é uma tarefa árdua que envolve várias nuances, a ser desenvolvido pelo gestor. Usar os tipos de liderança com suas determinadas características, pode ser necessário para envolvimento e respeito por parte da equipe. Deste modo, é observado nos relatos a seguir:

"O fato de não precisar gritar com eles, pra que eles possam me ouvir, o fato de trazer ele para perto de mim e quando preciso sempre estão a me ajudar. "(E-9)

"Eu tento persuadir, mostrar em números, percentual. Quando não consigo, tenho que exigir e quando não tem um consenso aí pronto é bater o martelo e segurar as pontas se caso não der certo. Mas devemos fazer isso vez enquanto". (E-5).

Os relatos evidenciam ainda que gestões autoritárias, que pautam em decisões unilaterais e impostas, não contribuem para engajamento da equipe, como pode-se observar abaixo:

"A gente tem que convencê-los você não pode impor; na verdade quando a gente precisa deliberar alguma coisa a gente vai explicar o porquê daquilo ali, a gente vai mostrar como a gente chegou naquela decisão e compartilhar com eles, e muitas vezes eles concordam, e eu compartilho muito os números da nossa unidade, o percentual das taxas de infecção, deixando os funcionários por dentro. Criar uma persuasão, explicar porque, persuadir a pensar da mesma forma que você”. (E-4)

"No trabalho devemos mostrar o profissionalismo e isso não pode ser esquecido, amigos e trabalho a parte, e na enfermagem nós temos essa dificuldade muito grande, as pessoas confundem muito isso. Respeitar para ser respeitado". (E-10)

Há características de liderança que são importantes fatores para contribuir no respeito e confiança da equipe que podem ser observados a seguir:

"A gente não é onipresente, mas percebo o respeito quando a gente, nas reuniões, a forma que eles dialogam com a minha pessoa, como eles executam as coisas que eu peço, então quando é que eu percebo que eu tenho respeito? Quando o que eu solicito é executado, quando eu não estou aqui e suas ações continuam da mesma forma, mesmo 
nível. As pessoas fazerem o que você orienta significa um respeito, que reconhece aquilo que você está falando, que é o melhor para equipe, é melhor para o serviço". (E-7)

"Acredito que se eu tenho resultados positivos, se a gente está alcançando e se agente reconhece a equipe, de certa forma a gente agrega valor àquela equipe e a equipe reconhece o valor que a gente tem. O líder geralmente vai ser considerado chato, é um pouco taxativo, mas quando o resultado chega e o resultado é alcançado e quando a gente compartilha isso com a equipe, isso é o mais importante". (E-7)

"Eu percebo pelo respeito que eles têm a mim, pela aproximação, eu sou muito de estar próximo a equipe, sempre gosto de estar na ponta, e eu gosto de gerenciar a gestão. Mas eu gosto de estar junto com eles, mas eu falho com a noite não podendo vir a noite. Mas já vim dar plantão a noite pra ficar mais próximo a eles”. (E2)

\subsection{Categoria III: Fatores que motivam os colaboradores no ambiente de trabalho}

A cultura da organização em que o método não punitivo é aplicado, favorece o desenvolvimento e a realização profissional. Observou-se nos depoimentos a busca constante pelo desenvolvimento de colaboradores de alto desempenho em detrimento da punição diante das falhas, como descrito:

"A gente sempre tem que estar estimulando, elogiando, reconhecendo pelas benfeitorias deles, parabenizando. Punir eu já não diria; hoje a gente tem uma visão de que a punição ela não é benéfica, ao contrário: a gente tem que trabalhar a fragilidade do colaborador, que levou a realizar algo, e ele crescer em cima do erro dele ou de outro da equipe; não punir, porque se eu o punir ele não vai se corrigir, ele vai se sentir punido e amanhã ele vai cometer, eu tenho que ensinar. Eu não sou a favor da punição por falha eu sou a favor de trabalhar a falha ". (E-6)

A valorização é fator determinante para melhores resultados no desmpenho das atividades, sendo descrito a seguir:

"Eu procuro respeitar todos, valorizar e a gente sabe qual é o colaborador que tem maior desempenho; esse colaborador de maior desempenho a gente dá algumas recompensas, que recompensas são essa? Por exemplo: às vezes estar precisando de uma folga de doação de sangue tal dia, mesmo sacrificando o serviço a gente sede a folga. A palavra punição não gosto dessa palavra, mas vamos dizer assim: conversar, dialogar, orientar, conduzir, direcionar e instruir e a palavra punir é muito pesada. Eu acredito que quando você orienta e instrui você mostra pra pessoa que aquela ação não foi legal, as vezes as pessoas fazem e não sabem que aquela ação não foi legal, então a pessoa aprende em cima daquele erro, mas tem que ser instruído, então eu dou um tempo, oportunidade, pra pessoa melhorar, aperfeiçoar-se". (E-3)

\subsection{Categoria IV: Fragilidade na formação acadêmica em gestão}

Nos discursos a seguir podemos ver a fragilidade, no que se refere, à formação acadêmica de enfermeiros em gestão, o que vem levando as instituições investirem em curso de pós-graduação em gestão de serviço em saúde.

“...quando saímos da academia nunca saímos preparados, o que nos prepara é o tempo, as experiências e a gente vai levando e vai começando a se sentir um pouco mais seguro diante de algumas ações; mas a academia na gestão, na gerência a gente sai pra aprender muito ainda, porque gerência de pessoas é o tempo que vai nos preparando”. (E6). 
Nota-se nos relatos que os profissionais necessitam de maior preparo na formação acadêmica, necessitando do desenvolvimento de habilidades de liderança:

\begin{abstract}
"A liderança é uma das principais características do enfermeiro, é o diferencial do enfermeiro e talvez seja a característica mais difícil; em algumas pessoas isso é nato, assim da pessoa mesmo. Mas para quem não tem, mesmo que tenha que desenvolver talvez seja muito difícil; para desenvolver isso talvez seja mais pela escuta, experiência, amadurecimento, vai se aprofundando, pensar sempre construtivamente, não ser taxativo com os funcionários, sempre pensar abertamente. Na vida acadêmica a gente é muito pouco preparado para liderança, muito pouco; a gente não é preparada para liderança se fala pouco em liderança, se estuda pouco sobre liderança; eu entendo também que é uma característica difícil de ser estudada, que a gente vai aos poucos aprimorando, mas é preciso; éo diferencial do enfermeiro; o enfermeiro é um líder de equipe ele não trabalha sozinho, então é preciso focar mais na
\end{abstract} liderança em enfermagem". (E-7).

\title{
4. Discussão
}

O enfermeiro desenvolve atividades gerenciais e assistenciais que envolvem processos gestores para produção do cuidado, tanto no gerenciamento dos serviços de saúde como da equipe de enfermagem, através de ações de planejamento, execução, coordenação, supervisão e avaliação (Metelski et al., 2020).

Além disso, envolve o desenvolvimento de competências gerenciais, como conjunto de conhecimentos, habilidades e atitudes que colaboram para o alto desempenho. Essas competências envolvem a tomada de decisão, comunicação, liderança, administração e gerenciamento, e educação permanente, que fomentam a formação crítico-reflexiva necessária para mudanças em saúde locais de médio e longo prazo (Ferracioli et al., 2020).

As entrevistas descritas destacam essas habilidades, como elementos para manutenção de qualidade nos serviços de saúde, potencializando engajamento da equipe de enfermagem juntamente a equipe de saúde para enfrentamento de problemas e melhoria da qualidade dos cuidados direcionados aos pacientes.

Pesquisas vem sendo desenvolvidas neste âmbito, especialmente envolvendo os modelos de liderança (Yi, 2018; Nanyonga et al., 2020; Bitencout et al., 2020; Moura et al., 2020; Ferracioli et al., 2020). Conhecimento para superação de disparidades de gênero em enfermeiros líderes na Coreia, assim como desafios para expansão e superação de problemas e transformação são destacados (Yi, 2018).

Do mesmo modo, situações como em Uganda em que o envolvimento de enfermeiros líderes em decisões em saúde são mínimos impactam em resultados em saúde. Aponta-se ainda que a cultura organizacional tem associação com práticas de liderança, e que mesmo em ambientes com poucos recursos é possível o desenvolvimento de liderança (Nanyonga et al., 2020).

No contexto pandêmico em que se vive atualmente, modelos de gestão e liderança estão sendo essenciais para remodelamento de instituições de saúde e acolhimento de pacientes. No Brasil, unidades especificas para pacientes com COVID-19 foram desenvolvidas por enfermeiros, com adaptações dos processos de enfermagem e ordenamento de novos de fluxos e rotinas. Além disso, atividades de dimensionamento de profissionais com experiência em cuidados críticos, liderança, gestão de riscos, e treinamentos foram destacadas, com ênfase na habilidade do enfermeiro para coordenação de elementos estruturais, recursos humanos e tecnológicos (Bitencout et al., 2020).

A formação do enfermeiro como líder envolve geralmente três processos singulares: as relações interpessoais desenvolvidas através de comunicação; o exercício da autonomia na resolução de conflitos, reconhecimento e respeito às individualidades dos componentes da equipe de saúde para alcance de objetivos comuns entre todos; e defesa da saúde, com respeito as singularidades e características de cada paciente para alcance de qualidade do cuidado (Pereira et al., 2021). 
Nos depoimentos descritos, a comunicação destaca-se como fator preponderante entre o enfermeiro como líder, sendo elemento essencial para se obter harmonia organizacional, satisfação e resolução de conflitos, assim como direcionamento dos componentes da equipe. Outros relatos destacam o respeito e o reconhecimento como estimulador positivo na equipe de enfermagem.

A comunicação contribui para equilíbrio no poder dentro da equipe de saúde, sendo ferramenta essencial do processo de trabalho, em que os componentes não sentem-se manipulados ou controlados mas colaboradores e cuidados pelo líder (Silva et al., 2017).

No entanto, observa-se carência de programas que fomentem a formação de líderes em instituições de saúde, além de profissionais que priorizem o gerenciamento do cuidado. Outra faceta encontra-se na deficiência de investimentos na formação de enfermeiros-líderes e na educação permanente (Amestoy et al., 2021).

Percebe-se isso nos relatos acerca das fragilidades na formação acadêmica em enfermagem direcionada à gestão e liderança. Amestoy et al. (2017) destaca ainda que a formação em liderança sofre influência da abordagem teórica e o âmbito hospitalar proporciona pouco suporte nos aspectos teórico e prático em liderança.

Evidencia-se atualmente que as melhores práticas de liderança envolvem competências técnicas e não técnicas como o desenvolvimento comportamental, conhecimento científico, diretrizes de qualidade e segurança do paciente, e gestão participativa da equipe (Silva et al., 2017). Modelos de liderança focados nestas competências são observados nos depoimentos, com utilização de evidências científicas direcionadas a melhoria da qualidade do cuidado aos pacientes, pautados em modelos de gestão participativa.

Do mesmo modo, modelos de liderança com colaboração, construção de confiança, auxiliando em relacionamentos interpessoais e desenvolvimento de competências dentro da equipe são destacados como modelos de liderança colaborativos que contribuem para mudanças na equipe de saúde. Neste modelo, comportamentos de dignidade e respeito, e relacionamento com os liderados e seu envolvimento são visíveis e desejáveis (Fusari et al., 2021).

Acerca de limitações no estudo, a principal envolveu conciliar horários de trabalho dos enfermeiros gestores com momentos de entrevistas, o que foi pactuado anteriormente e adaptado conforme rotina de cada setor da instituição de saúde.

Contribuições importantes sobre a liderança em equipes de enfermagem são visualizadas nos resultados, com grande impacto no desenvolvimento dos cuidados em instituições de saúde como construção de confiança e respeito na equipe, resolução de conflitos, melhoria no desempenho e na organização dos serviços de saúde. Investigar a liderança como elemento chave para melhoria das equipes de saúde e seus resultados é importante fator nos modelos de atenção em saúde e suscitam reflexões sobre elementos que influenciam como formação, construção de respeito, confiança, participação e colaboração.

\section{Conclusão}

O estudo possibilitou conhecer a percepção dos enfermeiros sobre liderança em enfermagem, bem como instrumento no seu processo de trabalho. Os participantes enfatizaram a adesão na prática por uma liderança pautada no diálogo, que representa a capacidade do líder de influenciar seus colaboradores a atuarem de maneira crítica e reflexiva, nas quais florescem oportunidades de trocas de conhecimentos e aprimoramento coletivo.

Salienta-se que independente da instituição hospitalar, os enfermeiros apresentaram dificuldades semelhantes, pontuando-se a dificuldade dos enfermeiros assistenciais em incorporar a liderança nos processos de trabalho, fator que pode estar relacionado ao convívio diário com os demais membros da equipe.

Ampliar as competências de liderança auxiliam na melhoria do desempenho da equipe de saúde, através de construção de confiança, comunicação adequada, atendimento de demandas dos profissionais da equipe, cooperação e valorização. 
Percebeu-se também que a formação acadêmica não prepara para liderança, mas com a experiência diária e a busca por novas formações, torna-se possível o desenvolvimento desta característica para os processos de cuidado e qualidade em saúde. São necessários novos estudos acerca da formação e suas influências na formação de enfermeiros líderes assim como nos diversos ambientes da enfermagem.

A liderança da enfermagem apresenta-se como essencial, sendo necessário qualificação, desenvolvimento de competências e habilidades especificas que propiciem harmonia organizacional, confiança da equipe e qualidade na assistência nas organizações de saúde.

\section{Referências}

Amestoy, S. C., Trindade, L. L., Silva, G. T. R., Santos, B. P., Reis, V. R. S. S., \& Ferreira, V. B. (2017). Liderança na enfermagem: do ensino ao exercício no ambiente hospitalar. Revista da Escola de Enfermagem Anna Nery, 21(4):e20160276.

Amestoy, S. C., Trindade, L. L., Silva, G. T. R., Martins, M. M., Varanda, P. A. G., \& Santos. I. A. R. (2021). Fragilidades e potencialidades na formação de enfermeiros líderes. Revista Gaúcha de Enfermagem, 42 (esp): e20200196.

Bitencourt, J. V. O. V., Meschial, W. C., Frizon, G., Biffi, P., Souza, J. B., \& Maestri, E. (2020). Protagonismo do enfermeiro na estruturação e gestão de uma unidade específica para COVID-19. Texto e Contexto Enfermagem, 29:e20200213.

Carrara, G. L. R., Bernardes, A., Balsanell,i A. P., Camelo, S. H. H., Gabriel, C. S., \& Zanetti, A. C. B. (2017). A utilização de instrumentos para avaliação da liderança nos serviços de saúde e enfermagem. Revista Gaúcha de Enfermagem, 38(3):e2016-0060.

Estalella, G. M., Zabaleguib, A., \& Guerrac, S. S. (2021). Gestión y liderazgo de los servicios de Enfermería em el plan de emergencia de la pandemia COVID-19: la experiencia del Hospital Clínic de Barcelona. Enfermería Clinica, 2021;31: 12-17.

Ferracioli, G. V., Oliveira, R. R., Souza, V. S., Teston, E. F., Varela, P. L. R., \& Costa, M. A. R. (2020). Competências gerenciais na perspectiva de enfermeiros do contexto hospitalar. Enfermagem em Foco, 11(1): 15-20.

Ferreira, G. E., Dall'Agnol, C. M., \& Porto, A. R. (2016). Repercussões da proatividade no gerenciamento do cuidado: Percepções de enfermeiros. Revista da Escola de Enfermagem Anna Nery, 20(3):e20160057.

Fusari, M. E. K., Meirelles, B. H. S., Lanzoni, G. M. M., \& Costa, V. T. (2021). Melhores praticas de liderança dos enfermeiros na gestao do risco hospitalar: estudo de caso. Revista Gaúcha de Enfermagem, 42 (esp): e20200194.

Metelski, F. K., Alves, T. F., Rosa, R., Santos, J. L. G., \& Andrade, S. R. (2020). Gestão do cuidado e o enfermeiro. Revista de Enfermagem UERJ, 28:e51457.

Minayo, M. C. S. (2014). O Desafio do Conhecimento: pesquisa qualitativa em saúde. (14a ed.), Hucitec.

Ministério da Saúde do Brasil. (2012). Resolução nº 466 do Conselho Nacional de Saúde, de 12 de dezembro de 2012 . Aprova as diretrizes e normas regulamentadoras de pesquisas envolvendo seres humanos. Diário Oficial da União, 13 jun 2013.

Moura, A. A., Hayashida, K. Y., Bernardes, A., Zanetti, A. C. B., \& Gabriel, C. S. (2019). Charismatic leadership among nursing professionals: an integrative review. Revista Brasileira de Enfermagem, 72 (Suppl 1): 315-320.

Moura, A. A., Bernardes, A., Balsanelli, A. P., Dessotte, C. A. M., Gabriel, C. S., \& Zanetti, A. C. B. (2020). Leadership and job satisfaction in the Mobile Emergency Care Service context. Revista Latino-Americana de Enfermagem, 28: e3260.

Nanyonga, R. C., Bosire, E. N., Heller, D. J., Bradley, E., \& Reynolds, N. R. (2020). Predictors of nursing leadership in Uganda: a cross-sectional study. Health Policy Planning, 35 (suppl 1):51-64.

Pereira, L. A., Barlem, E. L. D., Dalmolin, G. L., Rocha, L. P., Schalenberger, C. D., \& Hirsch, C. D. (2021). The process of becoming a head nurse: an ethnographic approach. Journal of Research Fundamental Care. Online, 13: 1-7.

Silva, V. L. S., Camelo, S. H. H., Soares, M. I., Resck, Z. M. R., Chaves, L. D. P., \& Santos, F. C. (2017). Leadership practices in hospital nursing: a self of manager nurses. Revista da Escola de Enfermagem da USP, 51:e03206.

Yi, M. (2018). Leadership in Nursing in Korea. Asian Pacific Island Nursing Journal, 3 (2): 56-64. 